\title{
Neodymium-doping Induced Superconductivity in 1111-SrFeAsF Iron-Pnictide
} System

\author{
S.V. Chong, ${ }^{\mathrm{a}, *}$ S. Hashimoto, ${ }^{\mathrm{b}}$ H. Yamaguchi, ${ }^{\mathrm{b}}$ K. Kadowaki ${ }^{\mathrm{b}}$ \\ ${ }^{a}$ Industrial Research Limited, P.O. Box31-310, Lower Hutt 5040, New Zealand \\ ${ }^{b}$ Institute of Materials Science and Graduate School of Pure \& Applied Sciences, University of Tsukuba, 1-1-1, \\ Tennodai, Tsukuba, Ibaraki 305-8573, Japan
}

\begin{abstract}
Polycrystalline $\mathrm{Sr}_{1-\mathrm{x}} \mathrm{Nd}_{\mathrm{x}} \mathrm{FeAsF}$ samples at various $\mathrm{Nd}$-doping levels were prepared using both stoichiometric mixture of the starting materials and in slight excess amount of FeAs in this study. Susceptibility and resistivity were studied down to 4 K showing superconductivity competing with a low-temperature magnetic ordering, and probably the coexistence of both well into the superconducting region. Temperature dependence of the resistivity of all the Nd-doped samples clearly show the presence of this transition below $15 \mathrm{~K}$, most likely originating from the magnetic ordering of Nd moments, being gradually attenuated but not completely suppressed leading into the optimum doping region at $\mathrm{x} \approx 0.54$. The spin-density wave anomaly at $175 \mathrm{~K}$ survives up to $0.35 \mathrm{Nd}$-doping, with superconductivity occurring only above $\mathrm{x}=0.40$. The maximum onset transition temperature, $T_{c}$, reaches as high as $54.3 \mathrm{~K}$ for $\mathrm{x}=0.70$, but superconductivity disappears beyond that. The estimated upper critical fields, $H_{\mathrm{c} 2}$, are high, with values exceeding 98 Tesla obtained from two samples of different doping level.
\end{abstract}

Keywords: A. Superconductors; D. Spin-density waves; D. Superconductivity

\footnotetext{
* Corresponding author. Tel.: +64-4-9313022; fax: +64-4-9313117.

E-mail address: s.chong@irl.cri.nz (S.V. Chong).
} 


\section{Introduction}

The discovery of superconductivity in iron-based compounds in early 2008 has generated a huge amount of research efforts in this field [1]. To date, up to five types of iron-based superconductors have been unveiled [2,3] including one being isostructural with the initially discovered iron oxypnictides ( $L n F e A s O$, where $L n=$ rare-earths), but with the oxygen constituent fully replaced by fluorine and the $L n$ site replaced by divalent cations to preserve charge neutrality. Similar to the FeAs-122 compounds, this so-called 1111-fluoroarsenide system comes in the form of SrFeAsF, CaFeAsF, BaFeAsF, and EuFeAsF [2-4/4-6]. Superconductivity can be induced upon the replacement of some of the divalent cations with trivalent rare-earth cations (La, $\mathrm{Pr}, \mathrm{Nd}$, and Sm) [4-7/6-9] or by substituting some of the Fe with Co in the iron-arsenide conducting layers $\left[{ }_{3} / 5,8 / 10\right]$. The maximum $T_{c}$ achieved in these compounds are only slightly higher than those of the corresponding rare-earth oxypnictides with the highest reported at $57.4 \mathrm{~K}$ in $\mathrm{Nd}$-doped $\mathrm{CaFeAsF}[6 / 8]$. In this paper, we report on the realization of superconductivity in Nd-doped SrFeAsF with a transition temperature of just above $54 \mathrm{~K}$. We also discovered on a unique way to vary the concentration of $\mathrm{Nd}$ in this quaternary system by adding fixed excess amount of FeAs which acts like a "solvent" in assisting the doping of $\mathrm{Nd}$ into the $[\mathrm{SrF}]^{+}$layers.

\section{Experimental}

Polycrystalline $\mathrm{Sr}_{1-\mathrm{x}} \mathrm{Nd}_{\mathrm{x}} \mathrm{FeAsF}$ samples were prepared by way of solid state reaction using $\mathrm{SrF}_{2}$, SrAs, NdAs, As and Fe as the starting materials. SrAs was pre-synthesized by reacting $\mathrm{Sr}$ metal pieces and As powder under low vacuum sealed inside a quartz 
tube at $600{ }^{\circ} \mathrm{C}$ for $10 \mathrm{~h}$. NdAs was prepared in a similar way but at a higher temperature of $900{ }^{\circ} \mathrm{C}$ for $15 \mathrm{~h}$. Two methods were employed to prepare the $\mathrm{Sr}_{1-\mathrm{x}} \mathrm{Nd}_{\mathrm{x}} \mathrm{FeAsF}$ samples - type I uses a “1:1:1:1” stoichiometric mixture of the starting materials, and type II uses a slight excess amount of FeAs according to the formula $\left(\mathrm{Sr}_{1-\mathrm{x}} \mathrm{Nd}_{\mathrm{x}} \mathrm{F}\right)_{20-2 \mathrm{y}}(\mathrm{FeAs})_{20+\mathrm{y}}$, where $y=2.5$ and 5 were used. This is equivalent to the addition of 0.75 and 1.5 moles of FeAs, respectively, into the unit formula $\left\{\mathrm{Sr}_{1-\mathrm{x}} \mathrm{Nd}_{\mathrm{x}} \mathrm{FeAsF}\right\}$. We found that superconducting samples are more readily prepared using type II method, while that of type I requires at least four-times repeated heating, grinding and pelletizing before evidence of superconductivity is observed. All samples were prepared by first heating the thoroughly ground mixed powder of the starting materials placed in sealed quartz tubes containing partially reduced argon gas at a rate of $50^{\circ} \mathrm{C} / \mathrm{h}$ to $1000{ }^{\circ} \mathrm{C}$ for $24 \mathrm{~h}$. The sintered products were then reground, pressed into a pellet and heated at $1000{ }^{\circ} \mathrm{C}$ for 20 to $30 \mathrm{~h}$ again under reduced argon gas atmosphere enclosed in a quartz tube - this last step was repeated for at least three times. During sample preparation following type II method, excess FeAs happened to leach out from the pellets after every sintering step. These FeAs granules, formed on the surface, were removed before the pellet was reground. Moreover, using this method and fixing the nominal value $\mathrm{x}$, in some cases we were able to vary the actual amount of Nd doped into SrFeAsF, which deviate from the nominal value by altering ' $y$ ' in the formula $\left(\mathrm{Sr}_{1-\mathrm{x}} \mathrm{Nd}_{\mathrm{x}} \mathrm{F}\right)_{20-2 \mathrm{y}}(\mathrm{FeAs})_{20+\mathrm{y}}$. The crystal structure of the resulting samples was characterized by powder x-ray diffraction (XRD) using $\mathrm{Cu} \mathrm{K} \alpha$ radiation. The concentration of Nd incorporated into the quaternary compound was determined by energy-dispersive x-ray spectroscopy (EDS). Susceptibility was measured in a Quantum Design (QD)-SQUID magnetometer, while 
the field dependent resistivity was carried out in a QD-PPMS with magnetic field up to $5 \mathrm{~T}$, and temperatures down to $4 \mathrm{~K}$.

\section{Results and discussion}

Fig. 1a shows the XRD patterns from three different $\mathrm{Sr}_{1-\mathrm{x}} \mathrm{Nd}_{\mathrm{x}} \mathrm{FeAsF}$ samples. The samples with $\mathrm{x}=0.06$ and 0.50 (real composition) were prepared using type I method from nominal $\mathrm{x}=0.1$ and 0.5 , respectively, while that of $\mathrm{x}=0.70$ was prepared using type II method, with nominal $\mathrm{x}=0.7$ and $\mathrm{y}=2.5$. At low $\mathrm{x}$ value, almost all of the Bragg's reflections can be indexed to the parent compound, SrFeAsF, based on the tetragonal ZrCuSiAs-type structure [4]. At higher x values, impurity phases such as $\mathrm{SrF}_{2}$, NdAs, FeAs and $\mathrm{Fe}_{2}$ As become dominant. Selected-area EDS analyses (in agreement with XRD) however do confirm grains/crystallites of the quaternary phase as well as the doping of Nd into SrFeAsF. Fig 1b-d show typical EDS spectra of the quaternary grains from the corresponding XRD samples. The inserted tables are average values of the "quaternary" constituents from the analysis of at least 10 sampling areas, which confirm the presence and the correct amount of the elements in the respective formula $\mathrm{Sr}_{1}$ ${ }_{x} \mathrm{Nd}_{\mathrm{x}} \mathrm{FeAsF}$. It should be pointed no oxygen-containing species were detected (within the resolution limit of EDS) from all our freshly prepared samples and those after magnetometry scans. Therefore, one can completely rule-out superconductivity coming from the presence of $\mathrm{NdFeAsO}_{1-\mathrm{x}} \mathrm{F}_{\mathrm{x}}$ or $\mathrm{NdFeAsO}_{1-\delta}$ secondary phases. A plot of the lattice parameters and cell volume versus Nd doping concentration from all samples is shown in the inset of Fig. 1a. The c-axis parameter remains unperturbed with increasing doping, but there is a slight increase in the a-axis lattice parameter above $0.4 \mathrm{Nd}$-doping. This is clearly manifested in the tetragonal unit cell volume, which also increases 
slightly to above $145 \AA^{3}$ at $x>0.4$, compared with SrFeAsF with a cell volume of 143 $\AA^{3}$. We are unsure at this point why these parameters do not decrease as in the case of rare-earth substituted SrFeAsF and CaFeAsF compounds [7-9], since $\mathrm{Nd}^{3+}(0.995 \AA)$ has a smaller ionic radius compared to $\mathrm{Sr}^{2+}(1.12 \AA)$ [11]. However, the change in value of these parameters in the present system is small, and the important point to stress here is the doping level at which this deviation occurs is actually correlated to the emergence of superconductivity as presented below.

The temperature dependent resistivity $(R-T)$ for a series of $\mathrm{Sr}_{1-\mathrm{x}} \mathrm{Nd}_{\mathrm{x}} \mathrm{FeAsF}$ samples measured using a standard four-probe dc method is shown in Fig. 2a. The displayed Nd compositions are averaged values obtained from EDS [12]. The resistivity traces of $\mathrm{x}=$ 0.06 and 0.23 decrease more rapidly below $175 \mathrm{~K}$ as the samples are cooled down. This is attributed to the magneto-structural transition due to the spin-density wave (SDW) instability which is also seen in all non-doped and low-doping SrFeAsF (not shown here) samples $[4,7,9]$. At the low temperature end, around $9 \mathrm{~K}$, another transition $\left(T_{\mathrm{N}}\right)$ is observed, which is most likely originating from the magnetic ordering of $\mathrm{Nd}$ ions observable in all our temperature dependent magnetization measurements $(M-T)$. Doping $\mathrm{Nd}_{\mathrm{x}}=0.36$ into SrFeAsF is sufficient to completely suppress the SDW anomaly at $175 \mathrm{~K}$ but no evidence of superconductivity is observed. This is quite surprising as normally this would be sufficient to induce superconductivity. A possible explanation is the low temperature transition (see inset) is still quite strong at this doping level where the resistivity rises sharply with decreasing temperature, peaking at around $11 \mathrm{~K}$ before decreasing again down to $4 \mathrm{~K}$. Upon $\mathrm{x}=0.43$ doping, a sharp drop in resistivity occurs, evidence of a superconducting transition with an onset $T_{c}$ commencing just above $43 \mathrm{~K}$. At higher Nd-doping ( $\mathrm{x} \geq 0.48)$, even higher onset $T_{c} s^{\prime}$ are realized reaching to a 
maximum value just above $54 \mathrm{~K}$ in $\mathrm{x}=0.70$. Interesting to note is the low temperature transition is still visible in the superconducting state, shifted to a higher temperature position at around $13.4 \mathrm{~K}$, but the magnitude of the transition itself is less pronounced compared with the non superconducting state. Also noticeable is the resistivity in $\mathrm{x}=$ 0.50 , prepared via type I method, has a high residual value $\sim 0.2 \mathrm{~m} \Omega \cdot \mathrm{cm}$, while those prepared in slight excess FeAs have residual resistivity close to zero perhaps as a result of better granular behavior due to the alloying process in $\left(\mathrm{Sr}_{1-\mathrm{x}} \mathrm{Nd}_{\mathrm{x}} \mathrm{F}\right)_{20-2 \mathrm{y}}(\mathrm{FeAs})_{20+y}$. As a result these alloyed samples also show higher superconducting volume fractions (see below). Beyond $x=0.70$, superconductivity vanishes and the low temperature magnetic transition becomes prominent again. While the origin of this magnetic transition is still under investigation, there is strong evidence to indicate it is also a competing factor to the already established competition between SDW and superconductivity. In the nonlanthanum rare-earth oxypnictides and $\mathrm{EuFe}_{2} \mathrm{As}_{2}$ superconductors, there exists similar low temperature antiferromagnetic transition of the rare-earth ions, which is suppressed into a short-range magnetic ordering, however, not completely destroyed upon doping $[13,14]$. Here we propose a similar scenario is occurring in $\mathrm{Sr}_{1-\mathrm{x}} \mathrm{Nd}_{\mathrm{x}} \mathrm{FeAsF}$. The results from the resistivity study are summarized in the doping phase diagram in Fig. 2b.

All the superconducting transitions are supported by $M-T$ measurements where both Meissner effect and magnetic screening are observed in the respective field-cooled and zero-field-cooled curves. Fig. 2c shows the $M-T$ data from all the superconducting $\mathrm{Sr}_{1-}$ ${ }_{x} \mathrm{Nd}_{\mathrm{x}} \mathrm{Fe}$ AsF samples. Due to the presence of large amount of impurities, the diamagnetic volume fraction is calculated to be around $10 \%$ from the zero-field-cooled traces of $\mathrm{x}=$ 0.43 and 0.54 , while that of $\mathrm{x}=0.70$ (sample with the highest $T_{c}$ ) is around $8 \%$. The susceptibility onset $T_{c}$ is at $40.9,47.1$ and $51.4 \mathrm{~K}$ for $\mathrm{x}=0.43,0.48$ and 0.50 , 
respectively; and 51.1, 52.1 and $53.1 \mathrm{~K}$ for the sample with $\mathrm{x}=0.54,0.64$ and 0.70 , respectively. The position of these transitions and their trend with doping is in good agreement with the resistivity result. Also noticeable is the low temperature magnetic ordering is the least pronounced in $\mathrm{x}=0.54$, which may indicate this doping is in the vicinity of the optimum-doping region in terms of the dominance of the superconducting state and the suppression of the competing magnetic orderings.

The critical current density loops that lie within grains $\left(J_{c}\right)$ are estimated from field dependent magnetization $(M-H)$ measurements at $6.5 \mathrm{~K}$ (lowest point in $M-T$ ) using the extended Bean Model [15] for $\mathrm{Sr}_{0.57} \mathrm{Nd}_{0.43} \mathrm{FeAsF}$ and $\mathrm{Sr}_{0.46} \mathrm{Nd}_{0.54} \mathrm{FeAsF}$, as both of these have the highest superconducting volume fraction and good composition homogeneity under EDS. Due to the presence of flux pinning the sign of the magnetization in the superconducting state depends on the direction of the sweeping field which resulted in the opening of the observed hysteresis loops between the ascending and descending branches of the magnetization as depicted in the $M-H$ curves (inset of Fig.3). A significant paramagnetic/ferromagnetic background superimposes on both the $M-H$ hysteresis loops due to the presence of Fe-based impurities as previously mentioned. Fig. 3 shows $J_{\mathrm{c}}$ in both samples are weakly field dependent and saturate in the mid $10^{5} \mathrm{~A} \mathrm{~cm}^{-}$ ${ }^{2}$ range at high fields with that of $\mathrm{Sr}_{0.46} \mathrm{Nd}_{0.54} \mathrm{FeAsF}$ slightly higher than in $\mathrm{Sr}_{0.57} \mathrm{Nd}_{0.43} \mathrm{FeAsF}$, perhaps an indication that $J_{\mathrm{C}}$ is weakly doping dependent in this 1111 compound. The self-field intragrain $J_{\mathrm{c}}$ in both compounds are just below $10^{6} \mathrm{~A} \mathrm{~cm}^{-2}$, which is comparable to those measured in the 1111-iron oxypnictides [16-18]. This again confirms the striking similarity between the rare-earth-doped 1111fluoroarsenides and their oxypnictide counterparts in terms of maximum $T_{c}, J_{\mathrm{c}}$ values, and one would expect a similarly high $H_{\mathrm{c} 2}$ and low anisotropy to follow suit. The upper 
critical field in both the above samples is determined from the temperature dependent resistivity under different magnetic fields (Fig. 4). Taking a criterion of $90 \%$ of the normal resistive as the reversible superconducting transition, and using the WerthamerHelfand-Hohenberg $(\mathrm{WHH})$ formula, $H_{\mathrm{c} 2}(0)=-0.693 T_{c}\left(\mathrm{dH}_{\mathrm{c} 2} / \mathrm{dT}\right)_{\mathrm{T}=T c}$, [19] the zero temperature upper critical field, $H_{\mathrm{c} 2}(0)$, is estimated to be 99 and 110 Tesla for $\mathrm{x}=0.43$ and 0.54 , respectively. There is an indication here that $H_{\mathrm{c} 2}$ may be higher in the compound close to optimum doping, but more experiments will have to be conducted to verify this and the similar behavior observed in the $J_{\mathrm{c}}$ measurements. In polycrystalline 1111 rare-earth oxypnictides, $H_{\mathrm{c} 2}$ from $50 \mathrm{~T}$ in $\mathrm{LaFeAsO}_{0.9} \mathrm{~F}_{0.1}$ up to as high as $230 \mathrm{~T}$ in $\mathrm{NdFeAsO}{ }_{0.82} \mathrm{~F}_{0.18}$ have been reported [20,21]. The values obtained for $\mathrm{Sr}_{1-\mathrm{x}} \mathrm{Nd}_{\mathrm{x}} \mathrm{FeAsF}$ in this study are also high and fall within the range of the oxypnictides, hence also pointing toward potential high-field applications for this material.

In summary, superconductivity using $\mathrm{Nd}$ as a dopant in $\mathrm{SrFeAsF}$ with maximum $T_{c}$ close to $54 \mathrm{~K}$ has been realized. All the superconducting samples show the complete suppression of the SDW anomaly at $175 \mathrm{~K}$, and the gradual diminishing of the lowtemperature magnetic transition in both $R-T$ and $M-T$ measurements leading up to optima-doping and reemerges thereafter. At intermediate doping level $(\mathrm{x}=0.36)$, even though the SDW anomaly is completely suppressed, this compound is not superconducting; the pronounced feature of the low temperature transition where a sharp increase in the resistivity cooling down from 16 to $11 \mathrm{~K}$ may be suppressing superconductivity. Superconductivity is only realized above $0.4 \mathrm{Nd}$-doping which is in line with the current findings in most rare-earth-doped 1111-fluoroarsenides where a high doping level is required. The critical current density of $\mathrm{Sr}_{1-\mathrm{x}} \mathrm{Nd}_{\mathrm{x}} \mathrm{FeAsF}$ measured at 
$6.5 \mathrm{~K}$ ranges from low $10^{6}$ to $10^{5} \mathrm{~A} \mathrm{~cm}^{-2}$, also accompanied by a high upper critical field with values exceeding $98 \mathrm{~T}$ being measured.

\section{Acknowledgments}

This work has been supported by JSPS-KAKENHI, Grant-in-Aid for Scientific Research (A) (18204031), the Ministry of Education, Culture, Sports, Science and Technology (MEXT), JAPAN, CREST JST, WPI at NIMS (MANA), JSPS Core-to-

Core Program - Strategic Research Networks, "Nanoscience and Engineering in Superconductivity (NES)”, and the JSPS postdoctoral fellowship.

\section{References}

[1] Y. Kamihara, T. Watanabe, M. Hirano, H. Hosono, J. Am. Chem. Soc. 130 (2008) 3296.

[2] K. Ishida, Y. Nakai, H. Hosono, J. Phys. Soc. Jpn. 78 (2009) 062001.

[3] H. Ogino, Y Matsumura, Y. Katsura, K. Ushiyama, S. Horii, K. Kishio, J. Shimoyama, Supercond. Sci. Technol. 22 (2009) 075008.

[4]F. Han, X. Zhu, G. Mu, P. Cheng, H.-H. Wen, Phys. Rev. B 78 (2008) 180503(R); M. Tegel, S. Johansson, V. Weiss, I. Schellenberg, W. Hermes, R. Pöttgen, D. Johrendt, Europhys. Lett. 84 (2008) 67007.

[5] S. Matsuishi, Y. Inoue, T. Nomura, H. Yanagi, M. Hirano, H. Hosono, J. Am. Chem. Soc. 130 (2008) 14428.

[6] G. Wu, Y. L. Xie, H. Chen, M. Zhong, R.H. Liu, B.C. Shi, Q.J. Li, X.F. Wang, T. Wu, Y.J. Yan, J.J.Ying, X.H. Chen, Chinese Sci. Bull. 54 (2009) 1872. 
[7] X. Zhu, F. Han, P. Cheng, G. Mu, B. Shen, H.-H. Wen, Europhys. Lett. 85 (2009) 17011.

[8] P. Cheng, B. Shen, G. Mu, X. Zhu, F. Han, B. Zeng, H.-H. Wen, Europhys. Lett. 85 (2009) 67003.

[9] G. Wu, Y.L. Xie, H. Chen, M. Zhong, R.H. Liu, B.C. Shi, Q.J. Li, X.F. Wang, T. Wu, Y.J. Yan, J.J.Ying, X.H. Chen, J.Phys.: Cond. Matter 21 (2009) 142203.

[10] T. Nomura, Y. Inoue, S. Matsuishi, M. Hirano, J.E. Kim, K. Kato, M. Takata, H. Hosono, Supercond. Sci. Technol. 22 (2009) 055016.

[11]R.C. Weast, M.J. Astle and W.H. Beyer (Eds), CRC Handbook of Chemistry and Physics, CRC Press, Cleveland, Ohio, 2007-2008.

[12] Samples with $x=0.06,0.19,0.23,0.30$ and 0.50 were prepared by type I method from nominal composition of $0.1,0.3,0.2,0.33$, and 0.5 , respectively. The rest of the samples with $\mathrm{x}=0.36,0.43,0.64,0.70$ and 0.79 were prepared from initial $\mathrm{Nd}$ nominal value of $0.4,0.5,0.6,0.7$ and 0.8 , respectively, and with $y=2.5$. Only sample with $\mathrm{x}=0.54$ was prepared from 0.6 nominal $\mathrm{Nd}$ and $\mathrm{y}=0.5$.

[13]L. Ding, C. He, J.K. Dong, T. Wu, R.H. Liu, X.H. Chen, S.Y. Li, Phys. Rev. B 77 (2008) 180510(R); P.J. Baker, S.R. Giblin, F.L. Pratt, R.H. Liu, G. Wu, X.H. Chen, M.J. Pitcher, D.R. Parker, S.J. Clarke, S.J. Blundell, New J. Phys. 11 (2009) 025010.

[14]H.S. Jeevan, Z. Hossain, D. Kasinathan, H. Rosner, C. Geibel, P. Gegenwart, Phys. Rev. B 78 (2008) 092406; A. Mitsuda, T. Matoba, H. Wada, arXiv: 0911.0862.

[15]C.P. Poole, Jr, in: C.P. Poole, Jr (Ed), Handbook of Superconductivity, Academic Press, San Diego, 2006, Ch. 4. 
[16]A. Yamamoto, A.A. Polyanskii, J. Jiang, F. Kametani, C. Tarantini, F. Hunte, J. Jaroszynski, E.E. Hellstrom, P.J. Lee, A. Gurevich, D.C. Larbalestier, Z.A. Ren, J. Yang, X.L. Dong, W. Lu, Z.X. Zhao, Supercond. Sci. Technol. 21 (2008) 095008.

[17]R. Prozorov, M.E. Tillman, E.D. Mun, P.C. Canfield, New J. Phys. 11 (2009) 035004.

[18] S.V. Chong, T. Mochiji, S. Sato, K. Kadowaki, J. Phys. Soc. Jpn.: Suppl. C 77 (2008) 27.

[19] N.R. Werthamer, E. Helfand, P.C. Hohenberg, Phys. Rev. 147 (1966) 295.

[20]X. Zhu, H. Yang, L. Fang, G. Mu, H.-H. Wen, Supercond. Sci. Technol. 21 (2008) 105001.

[21]X. Wang, S.R. Ghorbani, G. Peleckis, S. Dou, Adv. Mater. 21 (2009) 236. 
Figure captions:

Fig. 1. Powder $\mathrm{x}$-ray diffraction patterns of three selected $\mathrm{Sr}_{1-\mathrm{x}} \mathrm{Nd}_{\mathrm{x}} \mathrm{FeAsF}$ samples $(\mathrm{x}=$ 0.06, 0.50 and 0.70) (a), and the corresponding selected-area EDS spectra of the quaternary-phased grains (b) to (d).

Fig. 2. Temperature dependent resisitivity of a series of $\mathrm{Sr}_{1-\mathrm{x}} \mathrm{Nd}_{\mathrm{x}} \mathrm{FeAsF}$ samples (a). The resulting spin-density wave transition temperature, $T_{\mathrm{SDW}}$ ( $\boldsymbol{\square}$; left axis), low-temperature magnetic transition $T_{\mathrm{N}}\left(\diamond\right.$; right axis) and superconducting transition temperature $T_{\mathrm{c}}$ ( $\mathbf{\Delta}$; right axis) are plotted as a function of the "real" Nd-doping concentration in (b). (c) shows the mass susceptibility versus temperature of all the superconducting samples measured under 10 Oe applied field.

Fig. 3. Magnetic field dependence of the critical current density of $\mathrm{Sr}_{1-\mathrm{x}} \mathrm{Nd}_{\mathrm{x}} \mathrm{FeAsF}$ for $\mathrm{x}$ $=0.43$ and 0.54 . The inset shows the corresponding $M-H$ loops carried out at $6.5 \mathrm{~K}$.

Fig. 4. Temperature dependence of the upper critical field of $\mathrm{Sr}_{0.57} \mathrm{Nd}_{0.43} \mathrm{FeAsF}$ (left) and $\mathrm{Sr}_{0.46} \mathrm{Nd}_{0.54} \mathrm{FeAsF}$ (right). The insets show the corresponding $R-T$ in different magnetic fields with the dashed line indicating the extrapolation of the normal state resistivity. The two values in brackets are the calculated $\left(\mathrm{d} H_{\mathrm{c} 2} / \mathrm{dT}\right)$ slope for the determination of $H_{\mathrm{c} 2}(0)$. 
Figure 1
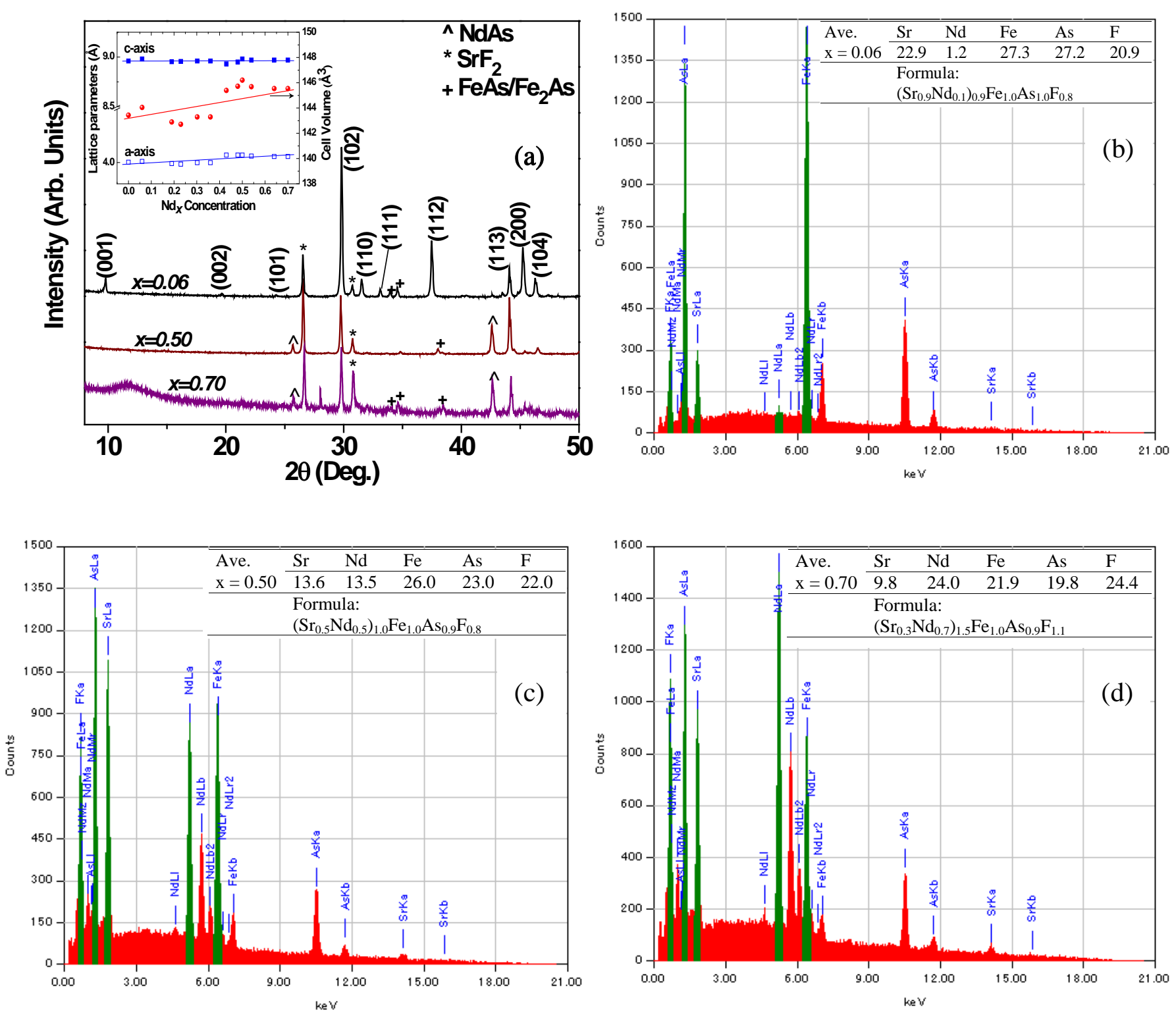
Figure 2

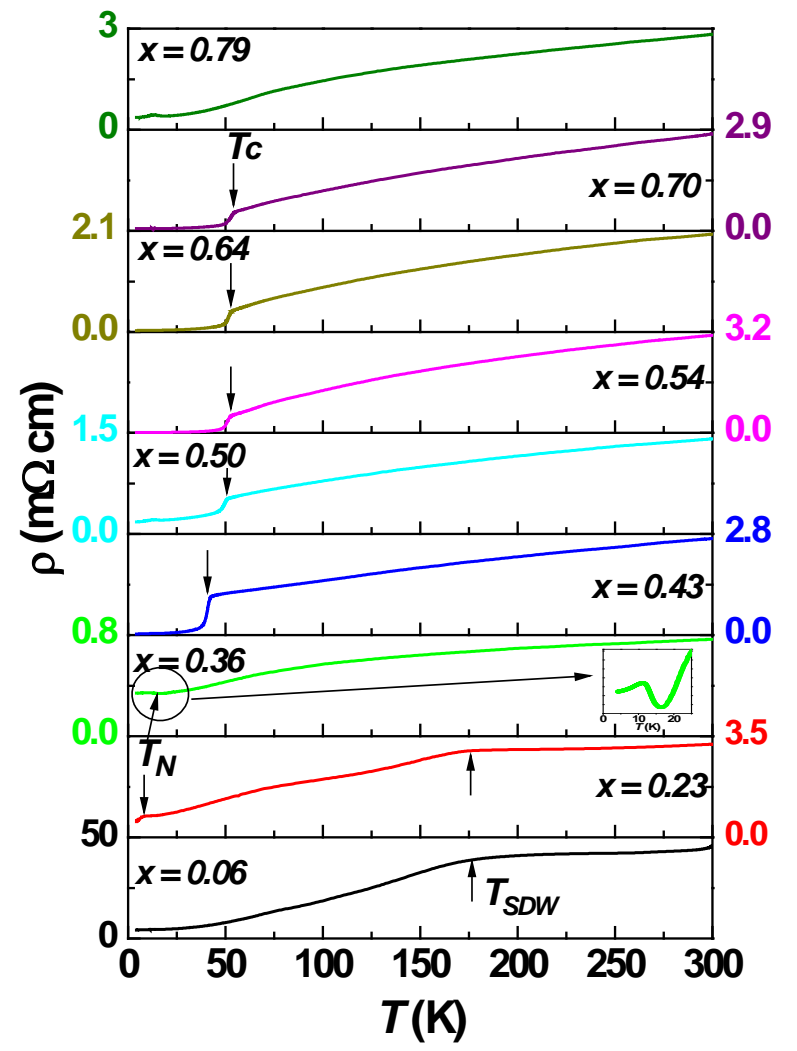

(a)

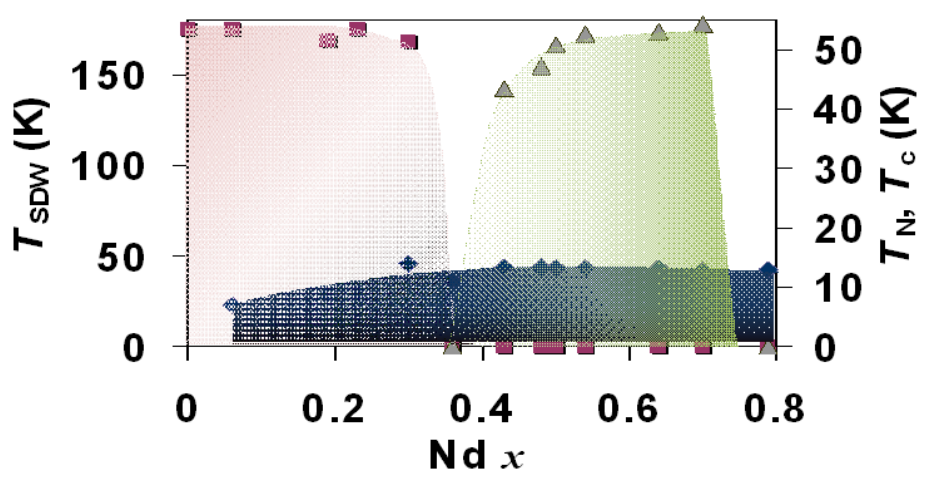

(b)

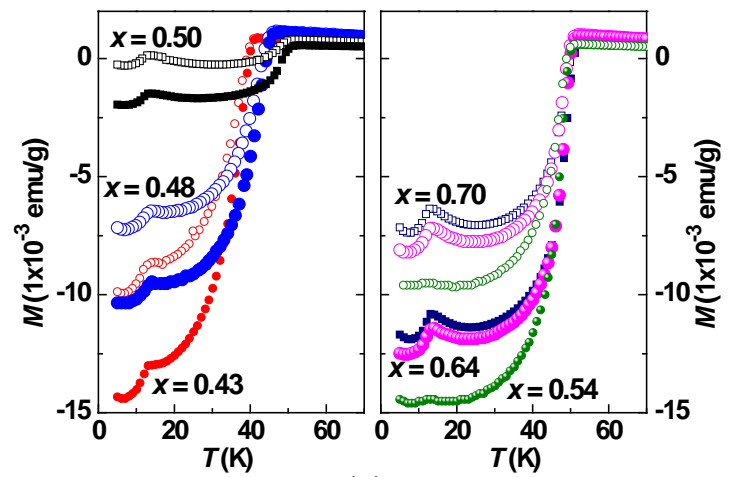

(c) 
Figure 3

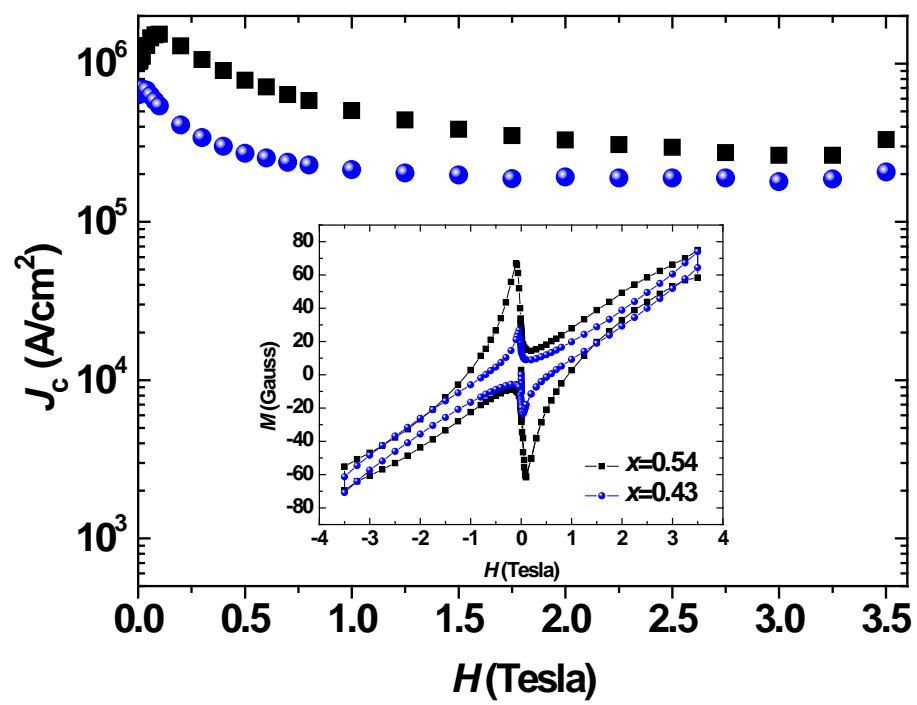


Figure 4

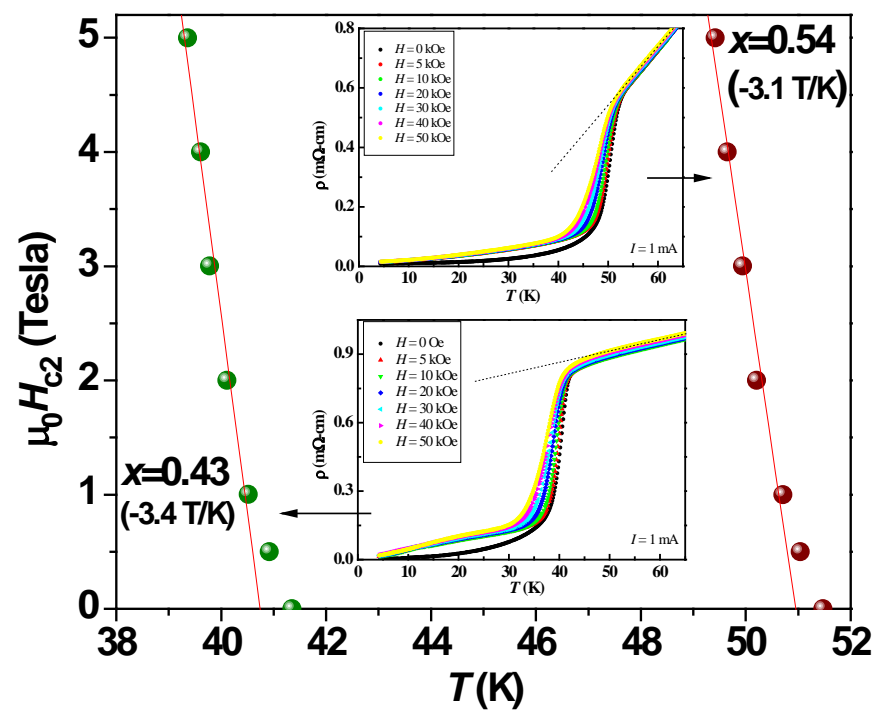

